\title{
OS NOVOS DESAFIOS DA ETNOGRAFIA: PARA ALÉM DA RESOLUÇÃO N 510/2016
}

\author{
RUI MASSATO HARAYAMA ${ }^{1}$
}

\begin{abstract}
RESUMO
Passados 20 anos da criação do Sistema CEP - Conep, apontamos novas configurações de tradicionais impasses éticos da pesquisa em Ciências Humanas, a saber: 0 processo de entrada em campo, a publicação dos resultados e 0 uso de dados obtidos na experiência profissional. A consolidação da lógica neoliberal da cultura das avaliações associada às novas legislações sobre o Consentimento Prévio, Livre e Informado, Acesso à Informação e Transparência, e sua interface com a profissionalização da antropologia e as novas ferramentas de obtenção da informação digital, colocam novas discussões sobre a ética em pesquisa não contempladas pela Resolução 510/2016. Por fim, ressaltamos a ausência de um controle social da pesquisa, que ainda permanece no campo ideal do Sistema CEP - Conep, mas que não é a realidade encontrada.
\end{abstract}

\section{PALAVRAS-CHAVE \\ Etnografia; Ética em Pesquisa; Culturas da Avaliação; Sistema CEP-Conep; Resolução $510 / 2016$}

\section{THE NEW CHALLENGES OFETHNOGRAPHY: BEYOND RESOLUTIONN ${ }^{\circ}$ 510/2016}

\begin{abstract}
In this article we highlight the new configuration of traditional ethical issues in Human Sciences Researches, after 20 years since CEP-Conep System creation. Namely the process of accessing the field, the publication of field results and the use of data obtained through professional experience. The Audit Culture's neoliberal logic associated with new legislations on Free and Informed Consent, Access to Information and Transparency, and its interface with anthropology professionalization and new tools for obtaining digital information, bring new Research Ethics issues not covered by Conep's Resolution 510/2016. Finally, we emphasize the absence of a social monitoring of, an ideal convention of the CEP-Conep System, but not a standard reality.
\end{abstract}

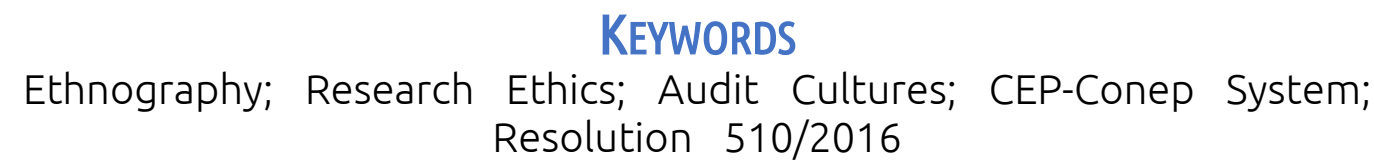

LOS NUEVOS DESAFÍOS DE LA ETNOGRAFÁA:MÁS ALLÁ DE LA RESOLUCIÓN N $510 / 2016$

\section{RESUMEN}

Después de 20 años de la creación del Sistema CEP-Conep, apuntamos hacia nuevas configuraciones de puntos muertos éticos tradicionales de la investigación en las Ciencias Humanas, como son: el proceso de entrada en campo, la publicación de los resultados y el uso de datos obtenidos en la experiencia profesional. La consolidación de la lógica neoliberal de la cultura de las evaluaciones asociada a las nuevas legislaciones sobre el Consentimiento Previo, Libre e Informado, Acceso a la Información y Transparencia y su interface con la profesionalización de la antropología y las nuevas herramientas de obtención de información digital, colocan

${ }^{1}$ Professor da Universidade Federal do Oeste do Pará. 
nuevas discusiones sobre la ética en investigación no contempladas por la Resolución 510/2016. Por último, resaltamos la ausencia de un control social de la investigación, que todavía permanece en el campo ideal del Sistema CEP-Conep, pero que no es la realidad encontrada.

\section{Palabras ClaVe \\ Etnografía; Ética en Investigación; Culturas de Evaluación; Sistema CEP-Conep; Resolución 510/2016}

\section{LES NOUVEAUX DÉFIS DE L 'ETHNOGRAPHIE: PAR-DELÀ DE LA RÉSOLUTION N 510/2016}

\section{RÉSUMÉ}

Se sont passés 20 ans de la création du Système CEP-Conep quand on objective des nouvelles configurations des impasses éthiques de la recherche au domaine des Sciences Humains. Autrement dit, les procédures pour faire l'entrée au terrain, la publication des résultats et l'usage des données obtenues dans l'expérience professionnelle. La consolidation de la logique néolibérale de culture des évaluations associées aux nouvelles législations sur le Consentement Préalable, Libre et Informé, Accès à l'Information et Transparence, et sa interface avec la professionnalisation de l'anthropologie et les outils nouveaux d'obtention des informations digitaux mettent en scène des nouvelles discussions sur l'éthique en recherche pas envisagées pour la Résolution 510/2016. Enfin, nous mettons en évidence l'absence du contrôle social de la recherche que se maintien au domaine de l'idéal du Système CEP-Conep, mais qu'en réalité n'est pas encore trouvé.

\section{MOTS-CLÉS}

Ethnographie ; Éthique de la recherche ; Culture des évaluations; Système CEPConep; Résolution 510/2016 


\begin{abstract}
Apesar dos fortes incentivos para pesquisas apoiadas pelo National Institute of Health (NIH) no Brasil, existem barreiras burocráticas significativas que afetam a pesquisa financiada pelo NIH. [...] $\mathrm{O}$ componente do processo de revisão que causa os atrasos mais longos está relacionado com a aprovação ética pela Conep. Todos os presentes na reunião NIHAABR (NIH Alumni Association of Brazil) expressaram preocupação não somente com os atrasos, mas também a falta de transparência no processo de aprovação e a imprevisibilidade dos resultados. [...] O novo Diretor de Cooperação Técnica de Ciência e Tecnologia do Ministério das Relações Exteriores (DCTEC), Ademar Seabra Cruz Junior, participou da reunião do NIHAABR e informou que esta foi a primeira vez que ele ouviu falar do problema. Indicou que esta era, em sua opinião, uma questão séria que prejudicava a necessidade de aumentar, e não impedir, a cooperação em saúde entre nossos países. Ele disse aos participantes que, ao retornar a Brasília, procuraria maneiras de começar a lidar com esse problema.
\end{abstract}

(Conferences Highlight Successes and Remaining Challenges in Health Cooperation Between the Us and Brazil2). $^{2}$.

Passados 20 anos de criação do Sistema CEP - Conep, a avaliação da nova Resolução n510/2016, específica para as Ciências Humanas, é um momento oportuno para realizarmos um panorama geral sobre a ética em pesquisa em ciências humanas e observar algumas dessas implicações que surgem reconfiguradas a partir de mudanças sociais nas duas últimas décadas, a saber: a questão do consentimento e autorização da realização da pesquisa e a preservação do sigilo da identidade dos interlocutores da pesquisa de campo.

Desde o início das discussões, antropólogos ressaltavam a inaplicabilidade do formato proposto pelos Comitês de Ética em Pesquisa, sobretudo no Termo de Consentimento Livre e Esclarecido e nas autorizações prévias de pesquisa. Luis Roberto Cardoso de Oliveira defendia a especificidade do processo de consentimento e autorização da pesquisa em Antropologia e dizia: "No caso da antropologia, a negociação da pesquisa e/ou do objeto é parte constitutiva do empreendimento: primeiro no campo e depois no escritório quando o trabalho é redigido, ainda que no segundo momento trate-se de um diálogo simulado." (OLIVEIRA, 2004, p. 35)

Esses dois momentos cruciais, a negociação da pesquisa - sua autorização e consentimento - e a publicação dos resultados da pesquisa, parecem ser os que mais trazem reflexões éticas ou ruídos com as populações pesquisadas. Entretanto, ao observarmos o modo como essas questões reaparecem em etnografias brasileiras contemporâneas, e balizadas pela discussão da Resolução $n^{\circ}$ 510/2016, faz-se necessário rever como se consolida o Sistema CEP - Conep no Brasil. Essa retomada histórica nos auxiliará a compreender esse sistema dentro de um escopo maior das agências de regulação e controle.

Foram nos dias 27 e 28 de Setembro de 1995, na 48 Reunião Ordinária do Conselho Nacional de Saúde (CNS), que o "Conselheiro Saad comentou que na primeira reunião em

\footnotetext{
2 Texto disponível em Public Library of US Diplomacy. Sítio eletrônico Wikileaks. Tradução minha. Disponível em: https://wikileaks.org/plusd/cables/09BRASILIA606 a.html. Acesso em: janeiro de 2017.
} 
que participou no CNS, opinou sobre a proposta de revisão da Resolução nº 01/88 e [que] do ponto de vista ético existem inovações a nível internacional." (CNS, 1995). É a partir da $48^{\circ}$ Reunião do CNS que se cria um Grupo de Trabalho para revisão da portaria que irá inscrever no cenário científico e social brasileiro os Comitês de Ética em Pesquisa (CEP) articulados pelo Conselho Nacional de Ética em Pesquisa (Conep), comumente chamado de Sistema CEP - Conep. A revisão pretendia criar uma nova resolução balizada pelos avanços mundiais e que extrapolasse os hospitais e as instituições de saúde universitários que realizavam pesquisas, para colocar o tema da ética em pesquisa para toda a sociedade.

Segundo William Saad Hossne, que presidiu o Grupo de Trabalho à época,

"Com base em dados dos Ministérios da Saúde, de Ciência e Tecnologia e da Educação, - grupo identificou cerca de trinta mil instituições universitárias, sociedades científicas, grupos de pesquisa, autoridades sanitárias, associações de portadores de deficiências etc. que, direta ou indiretamente, poderiam ter envolvimento com ética em pesquisa aos quais solicitou que enviassem sugestões para a elaboração da proposta. ${ }^{3 "}$ (HOSSNE, 2003, p. 65).

O envio dos ofícios com a consulta, associado aos seminários e palestras para a construção e validação do Sistema CEP - Conep, cristalizou-se na criação do seu mito de fundação: a Resolução 196/96 (PEREIRA, 2007).

Da sua promulgação até o presente momento, adensaram-se as publicações e debates que questionam ou justificam o modelo de controle da ética em pesquisa no Brasil. Esse percurso, desde a sua criação, também obteve sucesso na capilarização de um discurso que associa a ética em pesquisa à obtenção de um parecer de ética em pesquisa, ou seja, em um documento que viabiliza a publicação em livros e em periódicos, a obtenção de financiamentos, o acesso a terras indígenas ${ }^{4}$ e até mesmo mobiliza embaixadores e o Ministério das Relações Exteriores, como aponta o epílogo desse texto.

No entanto, desde a sua promulgação em 1996, já se tem o início dos questionamentos sobre a necessidade da adesão de pesquisadores das ciências humanas a esse sistema. É dessa época que se consolida a defesa da divisão das especificidades das pesquisas na área que seriam realizadas com seres humanos, ao passo que as pesquisas das ciências biomédicas seriam realizadas em seres humanos. Ou seja, na "pesquisa com seres humanos, diferentemente da pesquisa em seres humanos, o sujeito da pesquisa deixa a condição de cobaia (ou de objeto de intervenção) para assumir o papel de ator (ou de sujeito de interlocução)." (OLIVEIRA, 2004, p. 34).

\footnotetext{
${ }^{3}$ Em comunicação pessoal, Hossne me informou que o GT tinha enviado essa comunicação para a Associação Brasileira de Antropologia e demais instituições da pesquisa em Ciências Humanas.

${ }^{4}$ Ingresso em Terra Indígena. Sítio eletrônico FUNAl. Disponível em: http://www.funai.gov.br/index.php/servicos/ingresso-em-terra-indigena. Acesso em: janeiro de 2017.
} 
Essa divisão, que foi necessária e importante para pensarmos em mecanismos de diferenciação entre as pesquisas da área das ciências humanas daquelas da área das ciências biomédicas, encontrou um problema com a consolidação do modelo brasileiro. A análise realizada a partir dos dados dos projetos submetidos ao Sistema CEP - Conep, em 2006, demonstrou que a maioria dos projetos apresentados (69\%) eram das Ciências Humanas (HARDY, 2008). Esse fato demonstra que a simples divisão entre pesquisa das ciências humanas $x$ ciências biomédicas nem sempre reflete o contexto da prática científica brasileira, que é pautado pela disputa entre metodologias hardy e soft dentro da própria produção no campo da saúde, que abrange metodologias das ciências humanas e biomédicas. Um fato que pode ser justificado pelo consolidado campo interdisciplinar da Saúde Coletiva, assim como pelo uso, cada vez mais crescente, das pesquisas com metodologia qualitativa e não interventiva na área da saúde 5 .

Entretanto, tentar traçar a discussão sobre a ética em pesquisa no Brasil somente balizada pela história interna das discussões que envolvem o Sistema CEP - Conep, e sobretudo da Resolução $n^{\circ}$ 510/2016, seria criar um campo de discussão que se forma a reboque das exigências governamentais de regulação, como aponta Strathern (2006). Ou seja, se a discussão sobre a ética em pesquisa for somente balizada pelas discussões de documentos e resoluções que vêm sendo discutidos de forma tensa com a Conep, estaremos limitando a ética da pesquisa antropológica àquela preconizada pelo Sistema CEP - Conep e impediremos a promoção de um alargamento das necessárias reflexões sobre a ética em pesquisa. Assim como corremos o risco de não produzirmos o debate sobre os desafios em como criar mecanismos para minimizar os impactos negativos da prática da pesquisa nas populações estudadas, ou mesmo na criação de estratégias para a prática reflexiva do pesquisador na contemporaneidade. Afinal, é característico à antropologia a constante inserção da reflexão de seus procedimentos de pesquisa e pressupostos teóricos no modo como se produz conhecimento científico.

O início do Sistema CEP - Conep, a partir da análise documental e etnográfica (HARAYAMA, 2011; PEREIRA, 2007), aponta diretamente para a Resolução 196/96 como fator mítico da criação dos comitês de ética em pesquisa ${ }^{6}$. Esse fato, apesar de ser importante para a história institucional do Sistema CEP - Conep, precisa ser contextualizado

${ }^{5} \mathrm{O}$ debate sobre o uso de pesquisas não interventivas e a discussão ética pode ser aprofundada em Viegas, Harayama e Souza (2015).

${ }^{6}$ Não faz parte no mito fundante do Sistema CEP - Conep o ingresso, a partir da criação do sistema, de grandes empresas de Clinical Research Organizations (CROs), que começam a se instalar no Brasil com maior vigor, muitas das vezes adquirindo centros geridos por professores universitários, como é relatado em 'A Tale of Two CROs'. Sítio eletrônico ClinPage. Disponível em: http://www.clinpage.com/article/a tale_of two cros/print>. Publicado em 22/12/2008. Acesso em: janeiro de 2017. 
junto a outros fatos e eventos com o intuito de apontar pistas na compreensão do sucesso do Sistema CEP - Conep em se estabelecer em todo o Brasil. Para compreender a ascensão do sistema, o evento sociopolítico que mais chama atenção, na segunda metade da década de 1990, é a Reforma do Estado Brasileiro.

Essa reforma foi proposta durante o fim da crise monetário-inflacionária em 1995, com a instalação do Ministério da Administração Federal e Reforma do Estado (REPÚBLICA, 1995). A frente do Ministério, Bresser Pereira explica, em poucas linhas, o que é a reforma que transforma a administração pública burocrática em gerencial:

\begin{abstract}
"administração pública gerencial (tem como característica fundamental estar voltada para o cidadão-usuário [que] faz [o] controle através de resultados e não de procedimentos), neste tipo de administração é muito importante a separação clara do que é formulação de política e o que é execução de política." (BRESSER PEREIRA apud CNS, 1997, linha. 927-930, grifos nossos).
\end{abstract}

A reforma surge com a promessa de produzir um Estado virtuoso e eficiente, e se expressa na máxima da estadista Margareth Tatcher: There is no thing like society ${ }^{7}$.

Com esse jargão, Tatcher gera a desconstrução do imaginário da sociedade dentro das políticas públicas, argumento necessário para a proposta da reforma do estado inglês e a implementação de uma nova mentalidade de gestão: o NPM (New Public Management). A Nova Gestão Pública ${ }^{8}$ trouxe uma promessa: a modernização dos serviços públicos por meio da incorporação de elementos exitosos da gestão de mercado. E, no Brasil, a versão do NPM trouxe valores que, no linguajar cotidiano, são conhecidos como neoliberais: eficiência, tecnocracia, estado mínimo, qualidade, transparência, objetivos, resultados e accountability.

É dentro dessa nova configuração social, de um Estado sem sociedade, mas formado por homens, mulheres, professores, pesquisadores e demais indivíduos responsáveis e virtuosos, que surgem diversos mecanismos de accountability. O objetivo desses mecanismos é o de medir a performance individual e institucional, como o Sistema CEP Conep (criado em 1996), o Sistema de Avaliação CAPES (criado em 1996), a Plataforma Lattes (criada em 1999), o Exame Nacional do Ensino Médio ENEM (criado em 1998), entre outros sistemas de avaliação.

Esse fenômeno, que reverberou em diversos países, ficou conhecido como "Culturas da Avaliação" (Audit Cultures) (STRATHERN, 1999; 2000). Ou seja, é dentro desse cenário que podemos apontar os avanços e limites possibilitados pelo Sistema CEP - Conep: um sistema que mede a ética individual do pesquisador por meio de uma gestão do risco. Apesar de ser duramente criticado por pesquisadores que passam a chamá-los de 'burocracias da virtude' (JACOB; RILES, 2007), o modelo parece replicar o formato da obtenção de autorização e financiamento que regem as pesquisas em ciências humanas no mundo todo e que aliam

\footnotetext{
7 “Não há nada como a sociedade" (Tradução livre).

${ }^{8}$ Tradução livre para a sigla original NPM.
} 
mecanismos de accountability com a gestão de riscos. Aliás, é característica presente em diversos modelos de propostas de pesquisa itens como ética, gestão de risco e de impactos, categorias que muitas das vezes são utilizadas de forma procedimental por pesquisadores ${ }^{9}$.

Nesse panorama, a característica principal do Sistema CEP - Conep é a conjugação de um modelo de gestão do conhecimento, em nome da defesa do participante da pesquisa, intermediado pela estética da cultura da avaliação e da retórica da sociedade do risco. Uma tarefa que é realizada por meio de formulários, carimbos, assinaturas, declarações, autoavaliações e plataformas online de acesso público. E é dentro do arranjo contemporâneo dessas instituições neoliberais e da cultura da avaliação que se faz necessário discutir as implicações éticas para a prática antropológica, sobretudo nas mudanças significativas no modo como lidamos com a própria noção de conhecimento, informação e socialidade.

\section{ETNOGRAFIAS EM RISCO NO MUNDO DA INFORMAÇÃO DIGITAL}

Antropólogos são necessários porque eles trazem perspectivas únicas para a pesquisa de inteligência interdisciplinar. [...] Acreditamos, fortemente, que ao contribuir para uma melhor compreensão de culturas estrangeiras, antropólogos estariam contribuindo de forma positiva na formulação mais racional da política externa. Do nosso ponto de vista, tais contribuições são de interesse Nacional e coerentes com os preceitos éticos de sua associação. Esperamos que você concorde. (30 de Setembro de 1980, CIA). Carta de (apagado) рага (apagado) ${ }^{10}$.

A discussão sobre a ética da prática antropológica é inerente à própria ascensão da etnografia como metodologia privilegiada de pesquisa. Entretanto, desde a criação do Sistema CEP - Conep, o cenário brasileiro passou por transformações que mudaram de forma significativa a discussão sobre a prática e ética antropológica no Brasil.

Para além da retórica do risco da pesquisa e do controle social da pesquisa científica, implementados pelo Sistema CEP - Conep no marco jurídico, duas legislações surgem com grande impacto no questionamento de como atuamos em campo.

A primeira, o Decreto 5051 de 2004 (BRASIL, 2004), que promulga a Convenção no 169 da Organização Internacional do Trabalho (OIT) sobre Povos Indígenas e Tribais coloca,

\footnotetext{
${ }^{9}$ Em uma pesquisa internacional coordenada por mim em comunidades do Rio de Janeiro, foram necessárias diversas consultas jurídicas junto ao financiador americano para obter a dispensa de um seguro de vida para os participantes da pesquisa, esse item estava incluído nos itens de avaliação do risco. No entendimento dos financiadores, a avaliação de risco incluiria a contratação de um seguro de vida para os possíveis acidentes que poderiam ser causados pela participação na pesquisa, mesmo quando se tratasse de uma comunidade inteira. A justificativa, assim como em muitos processos de assinatura do Termo de Consentimento Livre e Esclarecido, estava baseada na proteção jurídica do patrocinador da pesquisa a eventuais processos futuros, ao invés da proteção dos participantes dela.
}

${ }^{10}$ Freedom of Information Act Electronic Reading Room CIA. Sítio eletrônico CIA. Disponível em: https://www.cia.gov/library/readingroom/document/cia-rdp86b00985r000100080020-2. Acesso em janeiro de 2017. 
por outras vias, a necessidade da Consulta Prévia, Livre e Informada para as ações legislativas e administrativas que possam afetar os territórios ou modos de vida de populações tradicionais. A inserção desse novo procedimento, apesar de previsto em outras legislações, como nas audiências públicas previstas na realização do Licenciamento Ambiental, criou uma tensão no que se considera válido como autorização e ação de pesquisa, muitas vezes colocando na berlinda antropólogos cuja prática é, ao mesmo tempo, acadêmica e indigenista. Afinal, onde tem fim a prática estritamente profissional antropológica e onde se inicia a prática de pesquisa? Com o aumento da atuação de antropólogos em diversas práticas profissionais, sobretudo egressos dos cursos de graduação e do mestrado, adensa-se a discussão sobre a interface entre ética profissional e ética em pesquisa. Em que medida, ao tratar de populações ou casos específicos encontrados em sua prática profissional, poderiam os antropólogos dizer que sua pesquisa não necessitaria de revisão ética, como aponta a Resolução $n^{\circ}$ 510/2016 no item "VII- pesquisa que objetiva o aprofundamento teórico de situações que emergem espontânea e contingencialmente na prática profissional, desde que não revelem dados que possam identificar o sujeito".

Com a expansão da atuação profissional do antropólogo, parece ser esse local entre pesquisador e profissional que coloca tensões e implicações éticas. Contendas recentes expõe essas nuances sob diversos ângulos. De um lado, podemos observar o caso dos Mundurukus que acusaram um antropólogo de não ter informado que estaria realizando pesquisa no momento em que trabalhava com uma organização indigenista. E em carta aberta informaram:

Toda e qualquer informação coletada sobre nós, não poderá virar dados de pesquisas que interessam ao empreendimento ou simples carreirismo acadêmico. Alguém usando indevidamente o nosso nome, nossa história, nossa cultura, esse alguém responderá pelos seus próprios atos e será responsabilizado por suas ações. (MOVIMENTO MUNDURUKU IPEREG'AYU, 2015)

E, de outro, podemos perceber que é somente trabalhando para instituições governamentais que se pode ter acesso a importantes discussões no que tange os direitos das populações indígenas, como pode ser observado na etnografia multissituada de Roberta Aguiar Cerri Reis, ao fazer uso de insights a partir da sua inserção como servidora pública em reuniões que ocorreram no contexto de implantação da hidrelétrica de Belo Monte (REIS, 2015).

Essa tensão também fica expressa na Proposta urgente $n^{0} 19$ do Eixo 6 - Direito à Memória e à Verdade apresentada na I Conferência Nacional de Política Indigenista que solicita

[...] incluir os autores indígenas como co-autores em todos os projetos de pesquisa ou outros projetos realizados com indígenas (recomendação expressa à Associação Brasileira de Antropologia ABA), garantindo que a memória e a história indígenas sejam contadas pelos próprios autores indígenas; bem como assegurando que as instituições de ensino e pesquisa requeiram maior comprometimento e ética dos 
pesquisadores em área indígena com a verdade, garantindo que os povos indígenas sejam consultados e deliberem quanto à realização da pesquisa com a garantia de um retorno, repartição dos benefícios oriundos das pesquisas e, nos casos em que a pesquisa for realizada sem autorização, sejam criados meios para denúncia e reparação. (FUNAl, 2016, p. 02).

Se muitos impasses e demandas estão relacionados aos usos dos dados e inserção política do pesquisador no contexto da atuação junto a povos indígenas, não se pode deixar de citar a carta do Movimento Mães de Maio (2015) que denuncia a prática de pesquisadores que se aproximam de movimentos sociais com o único objetivo de produzir conhecimento para ascender na carreira acadêmica.

Apesar dessa 'revolta do nativo' ser conhecida da antropologia, esse parece ser um ponto importante para pensarmos na ética em pesquisa, já que implica muitas pesquisas tradicionalmente realizadas por antropólogos e que não conseguem ser delimitadas pelos pressupostos da Resolução $n^{\circ}$ 510/2016, que segue no pressuposto da Res. 196/96 e da sua atualização 466/12, que implica que as pesquisas só possam ser iniciadas após avaliação de um Comitê de Ética em Pesquisa.

É urgente, sobretudo nas pesquisas realizadas na interface com o sistema oficial de saúde e de políticas públicas, uma reflexão sobre o uso de dados e de experiências profissionais para a produção de pesquisas na área das ciências humanas. Afinal, muitas das vezes, é a partir do acúmulo de experiências em campo, em momentos em que não nos enunciamos como pesquisadores, que nos fornecem insights para criar estratégias de, inclusive, preservar a identidade dos interlocutores. Não por um acaso, é recorrente a estratégia de criar cenas fictícias, mas que descrevem as dinâmicas das relações, em etnografias que lidam com temas sensíveis (BRONZ, 2016; COLEMAN, 2014; HARAYAMA, 2011) e que demonstram o caráter compreensivo do conhecimento antropológico, e não necessariamente memorialista e cartorial.

Essa discussão sobre o sigilo, como mostra Claudia Fonseca (2010), tangencia a própria discussão da função social e impacto político das pesquisas antropológicas. E alia-se à discussão do conhecimento antropológico, obtido por meio da etnografia, como forma objetiva de assegurar direitos de populações tradicionais.

Esse é um debate que tem tomado grande parte das discussões no meio antropológico e resulta, sobretudo, do aumento da inserção de antropólogos no meio profissional e na discussão sobre a ética na produção de laudos, diagnósticos e perícias. Um ponto importante, uma vez que na Resolução $n^{\circ}$ 510/2016, especificamente no item XV do parágrafo único, descreve-se a "pesquisa encoberta: pesquisa conduzida sem que os participantes sejam informados sobre objetivos e procedimentos do estudo, e sem que seu consentimento seja obtido previamente ou durante a realização da pesquisa". 
Apesar desse parecer um avanço, não se pode deixar de sublinhar o descompasso que esse item significa frente ao acúmulo das discussões sobre a produção do conhecimento científico antropológico como sendo dialogado com os interlocutores em campo, por meio da negociação da identidade do pesquisador, assim como nos vários exemplos de absorção do pesquisador dentro da rede de relações. Em outros termos, a inserção do pesquisador como parente, professor, doutor, profissional da saúde, jornalista, escritor, entre outros. Afinal, a imagem de um conhecimento positivo que pode ser acessado de forma mais pura e verdadeira, quando não se evidencia os objetivos da pesquisa e a identidade do pesquisador, parece estar aliado a um pressuposto ocidental de verdade, que antropólogos tradicionalmente tendem a questionar, inclusive em processos de perícia, como aponta o Protocolo de Brasília (ABA, 2015).

A segunda legislação recente que impacta diretamente nesses dois momentos importantes da pesquisa antropológica, a entrada em campo e a descrição dos resultados, é a Lei de Acesso à Informação (BRASIL, 2011), promulgada em 2011. Com esse marco jurídico, que caracteriza como direito fundamental do cidadão o acesso à informação, cria-se uma nova fronteira do que se considera sigiloso e público. As informações públicas, mesmo que sensíveis, não passam pela discussão dos Comitês de Ética em Pesquisa. Entretanto, são essas que geram discussões éticas, sobretudo quando se tem o cruzamento de dados de campo e documentos públicos (CUNHA; RIFIOTIS, 2016). Afinal, o pressuposto da transparência institucional, na qual a Lei de Acesso à Informação se ancora, faz parte dessa cultura da avaliação que prezam instituições transparentes e mais accountables, à medida que elas publicam dados. Entretanto, esse excesso de dados publicados, em nome de uma 'ditadura' da transparência, nem sempre são lidos, ou são publicados para serem lidos (CADUFF, 2017; STRATHERN, 2000b).

É nesse ponto que surge um impasse ético da pesquisa antropológica nesses casos: evidenciar e descrever nomes e situações, coletados entre inúmeros documentos publicizados, não necessariamente atesta a objetividade científica antropológica; por vezes, acaba gerando um aspecto cartorial e quase jurídico das relações observadas em campo, ou implica na estigmatização de populações e grupos sociais. Essa discussão, inclusive, tensiona o papel ético e político antropológico que essa nova economia do sigilo parece implicar: estamos etnografando padrões, culturas e relações ou procurando verdades ocultas?

Esse parece ser um problema catalisado pelas legislações de transparência, tendo em vista que pesquisadores começam a se perguntar como produzir uma economia descritiva que não seja acusatória e que possa descrever as formas como as relações ocorreram no campo observado. Passa a ser mais recorrente, entre pesquisadores, os questionamentos de como a sua pesquisa poderá ser utilizada, seja pelas populações estudadas, ou pelo poder executivo e legislativo. Alguns trabalhos utilizam-se de consultas 
informais a advogados que leem as etnografias para apontar possibilidades de processos judiciais futuros ${ }^{11}$, outros informam textos em jornais e documentos oficiais para corroborar as informações descritas com pseudônimos para que possam ser verificados. Essa estratégia de prever como as pesquisas podem ser utilizadas e contestadas torna-se comum, sobretudo pela facilidade com que as pesquisas passam a ser acessadas na Plataforma Sucupira ${ }^{12}$ por qualquer pessoa com acesso a internet.

O que essas duas legislações - OIT 169 e Lei da Transparência - apontam é para esse novo, e recorrente, questionamento antropológico: como se dá o processo de entrada em campo, dos pedidos de consentimento, e como se apresentam os dados e resultados.

Ainda que possamos verificar avanços da Resolução $n^{\circ}$ 510/2016 no que concerne ao Processo de Consentimento, que não precisa ser comprovado em formulários e pode ser obtido em qualquer fase da execução da pesquisa (conforme o Art. $4^{\circ}$ ), os mais de vinte anos de existência do Sistema CEP - Conep, não contemplou a discussão de outros modelos de Consentimento Informado que extrapolam o jurídico-cartorial. Mesmo Comitês de Ética em Pesquisas exclusivas para as Ciências Humanas, como é o caso do CEP/IH-UNB, disponibilizam modelos para serem impressos e assinados ${ }^{13}$. Dentro das pesquisas clínicas, o modelo do TCLE é constantemente posto em discussão por pesquisadores que demonstram que são as relações de confiança com a instituição da saúde, ou ainda, o acesso ao tratamento médico que garantem participação na pesquisa. Dado que é agravado pela baixa escolaridade e renda de grande parte dos participantes (LOBATO; GAZZINELLI, et al., 2014; MENEGUIN; ZOBOLI, et al., 2010). Esse é um ponto que precisa ser ressaltado e pensado de forma crítica por pesquisadores de ciências humanas. Afinal, o que motiva um grupo a aceitar ser etnografado e pesquisado? Fazendo um paralelo com as pesquisas clínicas onde os participantes estão em busca de tratamento médico, as pesquisas em antropologia estariam sendo aceitas com algum outro objetivo em mente? Se as intenções dos interlocutores ao participar de pesquisas não podem ser facilmente controladas, etnografias demonstram como a entrada do antropólogo está sendo interpretada como uma forma de acesso a laudos e defesa de direitos sociais (POUGET, 2010).

Nesse ponto, parece-nos que o desafio do Processo de Consentimento posto na Resolução $n^{\circ}$ 510/2016 se depara com dilemas já existentes sobre a Consulta Prévia, Livre e ${ }^{11}$ Essa prática foi comentada diretamente a mim por antropólogos, para preservar a identidade dos
pesquisadores não irei mencionar o nome dos trabalhos.

${ }^{12}$ A plataforma Sucupira foi criada com o objetivo de, entre outros, possibilitar "Maior transparência dos dados para toda a comunidade acadêmica" e está disponível em: http://capes.gov.br/avaliacao/plataforma-sucupira. Acesso em: janeiro de 2017.

${ }^{13}$ Comitê de Ética em Pesquisa em Ciências Humanas e Sociais Universidade de Brasília. Disponível em: http://www.cepih.org.br/pesquisador.htm. Acesso em: janeiro de 2017. 
Informada (CPLI) exigida pela OIT 169. Afinal, muitas das vezes, tanto no CPLI quanto no TCLE, o procedimento é realizado de forma estritamente burocrática e não permite o tempo suficiente para a discussão entre a população consultada. Não se pode deixar de notar que a própria regulamentação da CPLI sofreu impasses no caso brasileiro, ainda não equacionados. De um lado, a própria concepção do que se entende sobre povos indígenas e, de outro, a negação dos povos tradicionais ao direito de serem consultados a partir dessa legislação. Esse debate expõe a realidade de tutela presente em toda a política pública brasileira que não absorveu o pressuposto da autonomia das populações indígenas e tradicionais preconizada pela OIT 169 (REDE DE COOPERAÇÃO AMAZÔNICA - RCA, 2016). Afinal "a consulta prévia garante autonomia aos povos indígenas ou reproduz relações de colonialidade?" (OLIVEIRA; ALEIXO, 2014).

Os debates que se desenrolaram sobre o direito à oitiva e consulta prévia no licenciamento da construção da Usina de Belo Monte (BELTRÃO; OLIVEIRA; PONTES JR., 2014) demonstram como elementos da desigualdade social e racismo institucional se apresentam nesses processos para reiterar a assimetria das relações.

Рara além da difícil tarefa de dividir os momentos em que antropólogos estão executando atos de intervenção administrativa - como em projetos sociais, educacionais e de saúde -, dos momentos de pesquisa, a contenda sobre o CPLI coloca em evidência pressupostos relacionais assimétricos que, muitas das vezes, os processos de consulta e consentimento apenas reiteram. Afinal, como conseguir separar os momentos de intervenção social dos momentos de solicitação da autorização da pesquisa, como o caso dos Mundurukus evidencia? Ou ainda: como conseguir afirmar, no delicado processo de obtenção da autorização, que os objetivos da pesquisa não preveem a assessoria do pesquisador para a implementação de projetos culturais ou captação de recursos para a gestão das organizações que nos aproximamos quando realizamos pesquisa, como fica explícito na demanda do Movimento Mães de Maio?

Se essa é uma discussão presente em outros momentos da história da antropologia brasileira (DURHAM, 1986), com o avanço e massificação da internet, sobretudo nas redes móveis, e o surgimento do fenômeno das redes sociais, que tornam seus participantes em produtores ativos e imediatos no compartilhamento de informação, assim como o vazamento de dados como o Wikileaks, Anonymous e Panama Papers, novos questionamentos éticos surgem para os antropólogos: como iremos produzir e expor os dados de nossa pesquisa de campo; como iremos responder às demandas dos interlocutores de campo.

Afinal, por mais que haja empenho na escrita para garantir o sigilo dos interlocutores da pesquisa, muitas das vezes, o simples cruzamento de atas de reuniões locais e/ou governamentais, publicizados em Portais da Transparência, com a coleta de 
informações em redes sociais pode apontar o local e as pessoas relacionadas nos trabalhos de campo. Informações que podem ser usadas, inclusive, na judicialização de laudos e perícias. Assim como bastam algumas consultas para que os envolvidos na pesquisa de campo possam acessar os resultados, seja no formato de teses e dissertações, assim como em artigos e vídeos.

A ética da pesquisa em antropologia, nesse cenário de cultura das avaliações e extrema troca de informações, coloca novos questionamentos sobre como iremos negociar e firmar promessas na entrada da pesquisa de campo, assim como na produção e publicação dos resultados ${ }^{14}$.

Por fim, para ampliar o debate sobre a ética das pesquisas em ciências humanas, e se nos atermos ao pressuposto do modelo brasileiro, no qual a ética em pesquisa é pensada como inserção e controle da produção científica pela sociedade, poucos foram os avanços no chamado 'controle social' da pesquisa científica. A Plataforma Brasil, lançada em 2011 com a promessa de melhorar a relação entre pesquisadores, comitês de ética em pesquisa e a sociedade, encontra impasses com a lentidão na atualização de sua tecnologia para a inserção das pesquisas em ciências humanas, assim como não possui ferramenta clara para a consulta de pesquisas aprovadas pelos Comitês de Ética. Esse último ponto, que seria a parte do controle social via a transparência dos dados, caro a essas instituições de avaliação de riscos, ainda encontra-se aquém do desejado.

Como exemplo, uma pesquisa que realizou um levantamento da saúde mental em crianças e jovens quilombolas da llha de Maré, Salvador, por meio de um questionário não interventivo - o Strengths and Difficulties Questionnaire ${ }^{15}$ (SDQ) (GOODMAN; NEVES DOS SANTOS, 2005), que é citado em uma Meta-análise (POLANCZYK; SALUM, et al., 2015 ) como evidência intercultural da prevalência de doença mental em crianças e adolescentes, nos alerta sobre os dilemas éticos nas quais as pesquisas estão inseridas nos dias de hoje. Seria essa pesquisa, que apenas faz uso de questionários respondidos por pais e professores, avaliada como pesquisa da área de ciências humanas ${ }^{16}$ ? Esta pesquisa estaria garantindo que os resultados não estigmatizariam a população formada de crianças e jovens quilombolas? Essas dúvidas, entretanto, não são sanadas, uma vez que o projeto de pesquisa não se encontra disponível na Plataforma Brasil para ser consultado, assim como nenhum registro dessa pesquisa foi encontrado pela equipe da Conep, conforme protocolo

14 Em conversa informal, alguns pesquisadores revelaram não enviar trabalhos escritos para congressos quando ainda tinham dúvidas sobre as interpretações dos dados do campo com o receio de serem interpelados pelos nativos.

15 "Questionário de pontos fortes e dificuldades" (Tradução livre).

16 O questionário em português está disponível no site Youth in Mind, no link http://www.sdainfo.com/py/sdainfo/b3.py?language=Portugueseqz (Brazil). Acesso em: janeiro de 2017. 
25820000222201756. Afinal, como antropólogos, é importante lembrar que o questionamento da estigmatização de grupos, classes e etnias via teorias científicas sempre foi uma questão de ordem ética e política, que atravessou grande parte da história da produção das ciências humanas.

Nesse sentido, é importante reafirmar a discussão da ética em pesquisa para além da defesa das especificidades das pesquisas em ciências humanas, tendo em vista o campo plural que esse termo agrega. Assim como é necessário reafirmar a importância do debate sobre a própria concepção de ciência e humanidade que essas novas resoluções e sistema de avaliação permitem. Sem uma discussão sobre a ética em pesquisa e o papel do conhecimento científico nos dias de hoje, o acúmulo da participação da antropologia nos debates sobre a ética em pesquisa, na arena das resoluções do Sistema CEP - Conep, corre o perigo de ficar restrito a um grupo de pesquisadores especialistas no tema.

\section{REFERÊNCIAS}

ABA. Protocolo de Brasília: laudos antropológicos: condições para o exercício de um trabalho científico. Rio de Janeiro: ABA, 2015.

BELTRÃO, Jane Felipe; OLIVEIRA, Assis da Costa; PONTES JR., Felício. Significados do Direito à consulta: povos indígenas versus UHE Belo Monte. In: OLIVEIRA, J. P. D.; COHN, C. Belo Monte e a questão indígena. Brasília: ABA, 2014. p. 70-101.

BRASIL. DECRETO No 5.051, DE 19 DE ABRIL DE 2004. Disponível em: http://www.planalto.gov.br/ccivil 03/Ato2004-2006/2004/Decreto/D5051.htm. ed. Brasília: [s.n.], 2004. Acesso em: janeiro de 2017.

BRASIL. LEI No 12.527, DE 18 DE NOVEMBRO DE 2011. [S.l.]: [s.n.], 2011.

BRONZ, Deborah. Nos Bastidores do Licenciamento Ambiental.. Rio de Janeiro: Contra Capa, 2016.

CADUFF, Carlo. Targets in the Cloud: On Transparency and Other Shadow. Science, Technology \& Human Values, 2017. 1-5. DOI: 10.1177/0162243916687644

CNS. ATA 48, Brasília, 27 e 28 Setembro 1995. Disponível em: http://conselho.saude.gov.br/atas/1995/ata48.DOC.

CNS. ATA 64, Brasília, 2/3 Abril 1997a. Disponível em: http://conselho.saude.gov.br/atas/1997/ata64.doc.

COLEMAN, Gabriella. Hacker, Hoaxer, Whistleblower, Spy: The Many Faces of Anonymous. Londres: Verso, 2014. S91-S102, 2017.

. From Internet Farming to Weapons of the Geek. Current Anthropology, v. 58, n. 15, p. 
CUNHA, Patricia Marcondes Amaral da; RIFIOTIS, Theóphilos. "A publicidade como regra e o sigilo como exceção": A publicização online de acórdãos referentes à "violência sexual" e os desafios para a etnografia de documentos. Anais da 30aRBA. [S.l.]: ABA. 2016. Disponível em: http://www.30rba.abant.org.br/simposio/view?ID_MODALIDADE TRABALHO=2\&ID SIMPOSIO=9.

Acesso em: janeiro de 2017.

DURHAM, Eunice. Ribeiro. Pesquisa Antropológica com populações urbanas: problemas e perspectivas. In: CARDOSO, R.(Org.) A Aventura Antropológica. Rio de Janeiro: Paz e Terra, 1986. p.17-38.

FONSECA, Claudia. 0 anonimato e 0 texto antropológico: dilemas éticos e políticos da etnografia 'em casa'. In: SCHUCH, P.; VIEIRA, M. S.; PETERS, M. (Org.). Experiências, dilemas e desafios do fazer etnográfico contemporâneo. Porto Alegre: Ed. UFRGS, 2010. p. 205-227.

FUNAI. I Conferência Nacional de Política Indigenista - Propostas priorizadas pela Plenária Final. FUNAI. $\quad$ Brasília. 2016.201 Disponível http://www.funai.gov.br/arquivos/conteudo/ascom/2016/doc/propostasurgentesnovo.pdf. Acesso em: janeiro de 2017.

GOODMAN, Robert et al. The Itha de Maré study: a survey of child mental health problems in a predominantly African-Brazilian rural community. Social Psychiatry Psychiatry Epidemiology, 40, p. 1117, 2005. DOl: 10.1007/s00127-005-0851-z

HARAYAMA, Rui Massato. Do ponto de vista do sujeito da pesquisa: evento e cultura material em um comitê de ética em pesquisa. 2011. Dissertação (Mestrado em Antropologia)-Faculdade de Filosofi a e Ciências Humanas, Universidade Federal de Minas Gerais, Belo Horizonte, 2011.

HARDY, Ellen. AVALIAÇÃO DO SISTEMA CEP/CONEP Relatório - Etapa 1. Campinas: CEMICAMP/ UNICAMP. Agosto 2008.

HOSSNE, William Saad. 0 poder e as injustiças nas pesquisas em seres humanos. Interface comunicação, saúde, educação, v.7, n.12, p.55-70, 2003.

JACOB, Marie-Andree.; RILES, Annelise. The New Bureaucracies of Virtue: Introduction. Political and Legal Anthropology Review, v. 30, n. 2, p. 181-191, 2007.

LOBATO, Lucas et al. Conhecimento e voluntariedade para participação em pesquisas: um estudo descritivo com participantes de um ensaio clínico. Cadernos de Saúde Pública, Rio de Janeiro, jun 2014. 1305-1314.

MENEGUIN, Silmara et al. Entendimento do Termo de Consentimento por Pacientes Partícipes em Pesquisas com Fármaco na Cardiologia. Arquivos Brasileiros de Cardiologia, v.94, n.1, p. 4-9, 2010.

MOVIMENTO INDEPENDENTE MÃES DE MAIO. Carreirismo Acadêmico, Ongueiro e Estatal. Sítio eletrônico Zagaia. Disponível em: http://zagaiaemrevista.com.br/article/carreirismo-academicoongueiro-e-estatal/\#sthash.s9wFY5AG.dpuf. Acesso em: janeiro de 2017.

MOVIMENTO MUNDURUKU IPEREG'AYU. Quem são os muitos Daniel Belick? Xingu Vivo, 11 maio 2015. Disponível em: http://xingu-vivo.blogspot.com.br/2015/05/quem-sao-0s-muito-daniel-belick.html. Acesso em: janeiro de 2017. 
OLIVEIRA, Luis Roberto Cardoso de. Pesquisa em Versus Pesquisa Com Seres Humanos. In: VICTÓRIA, C. et al. Antropologia e ética: 0 debate atual no Brasil. Rio de Janeiro: Ed. UFF, 2004. p. 33-44.

OLIVEIRA, Rodrigo Magalhães de; ALEIXO, Mariah Torres. Convenção 169 da OIT em disputa: consulta prévia, pensamento descolonial e autodeterminação dos povos indígenas.

Anais da $29^{a}$ Reunião Brasileira de Antropologia. [S.l.]: [s.n.]. 2014. Disponível em: http://www.29rba.abant.org.br/resources/anais/1/1402003900 ARQUIV0 Oliveira\&Aleixo29RBA-

GT.48.pdf. Acesso em: janeiro de 2017.

PEREIRA, Márcia dos Santos. Análise da Institucionalização do Comitê de Ética em Pesquisa da Universidade Federal de Minas Gerais (1997-2007) Complexidades e Desafios. Belo Horizonte: Tese (doutorado) - Universidade Federal de Minas Gerais. 2007.

POLANCZYK, Guilherme et al. Annual research review: A meta-analysis of the worldwide prevalence of mental disorders in children and adolescents. Journal Child Psychology Psychiatry, v.56, n.3 p.345-365, 2015. Dol: $10.1111 /$ jcpp. 12381

POUGET, Frederic Caires. Prática arqueológicas e alteridades indígenas. São Paulo: Dissertação de Mestrado Apresentada no MAE USP, 2010.

REDE DE COOPERAÇÃO AMAZÔNICA - RCA. DIFICULDADES E RESISTÊNCIAS no processo de implementação do direito à consulta livre, prévia e informada no Brasil. São Paulo: Rede de Cooperação Amazônica - RCA, 2016.

REIS, Roberta Aguiar. NÓS, OS OUTROS E OS "PARENTES": POLÍTICA E POVOS INDÍGENAS NO CONTEXTO DE IMPLANTAÇÃO DA HIDRELÉTRICA DE BELO MONTE. Brasília: Dissertação apresentada ao Programa de Pós-Graduação em Antropologia Social da Universidade de Brasília (DAN /UnB), 2015.

REPÚBLICA, P. D. Plano Diretor da Reforma do Aparelho do Estado. Brasília: MARE, 1995.

STRATHERN, Marilyn. 'Melhorar a Classificação': a avaliação no sistema universitário britânico. Novos Estudos CEBRAP, v. 53, p. 15-31, 1999.

London: Routledge, 2000.

Audit Cultures: Anthropological studies in accountability, ethics and the academy. Accountability. and ethnography. In: STRATHERN, M. Audit Cultures: Anthropological studies in accountability, ethics and the academy. London: Routledge, 2000b. p. 279-304.

Bullet-proofing: a tale from United Kingdom. In: RILES, A. Documents: Artifacts of Modern Knowledge. Ann Arbor: The University of Michigan Press, 2006. p. 181-205.

VICTORIA, Ceres et al. Antropologia e Ética: 0 debate atual no Brasil. Niterói: EdUFF, 2004.

VIEGAS, Lygia de Sousa; HARAYAMA, Rui Massato; SOUZA, Marilene Proença Rebello de. Apontamentos críticos sobre estigma e medicalização à luz da psicologia e da antropologia. Ciência e saúde coletiva, v. 20, n. 9, p. 2683-2692, 2015. 